Revista Matemática Universitária, vol. 1, 2019

ISSN: 2675-5254 - DOI: https://doi.org/10.21711/26755254/rmu202014

\title{
RESOLVENDO RECORRÊNCIAS
}

\author{
ANTONIO CAMINHA
}

\begin{abstract}
Resumo. O problema de obtenção de fórmulas posicionais para sequências recorrentes é de importância central em Combinatória. Este artigo mostra como a teoria elementar de séries de potências complexas pode ser utilizada para resolvê-lo. Primeiro, no caso mais simples de recorrências lineares de coeficientes constantes, depois, em um caso não linear combinatorialmente relevante.
\end{abstract}

\section{INTRODUÇÃO}

Em Combinatória, é bastante comum que a modelagem analítica de problemas recursivos traduza-se no problema de obter fórmulas posicionais para sequências que satisfaçam relações de recorrência. Nesse sentido, cursos introdutórios de Matemática Discreta ou Combinatória gastam tempo considerável apresentando técnicas para a resolução de tais recorrências, o mais das vezes apoiando-se no uso de séries de potências formais.

Em que pese o fato de a correção do uso de séries formais poder ser justificada de maneira relativamente simples (cf. [5], por exemplo), esse ponto não é usualmente apresentado, ou mesmo mencionado, aos estudantes. Por outro lado, mesmo quando outras disciplinas de um curso de Licenciatura ou Bacharelado em Matemática ou Computação ocupam algum tempo com o tema, usualmente o fazem somente para recorrências lineares de coeficientes constantes e no contexto de tópicos bem mais avançados, por exemplo o teorema da decomposição primária em Álgebra Linear.

Uma vez que recorrências de coeficientes analíticos podem ser vistas como análogos discretos da classe correspondente de equações diferenciais, espera-se que a teoria de séries de potências complexas possa ser aplicada com sucesso em seu estudo. Esta nota pretende mostrar que esse é de fato o caso, de sorte que o tema passe a ser acessível a estudantes que tenham sido apresentados à teoria básica de séries de potências, conforme desenvolvida no Capítulo 11 de [1], por exemplo.

Por simplicidade, começaremos analisando o caso de recorrências lineares de coeficientes constantes, mostrando no Teorema 2.1 como resolvê-las rigorosamente;

Data de aceitação: 30 de setembro de 2019. 
em seguida, aplicamos esse resultado à análise de progressões aritméticas de ordem superior.

No que concerne o caso não linear, mostramos como argumentos de análise real elementar, juntamente com a teoria de funções de uma variável complexa fornecem uma demonstração simples (e, tanto quanto saibamos, nova) da clássica fórmula de inversão lagrangiana. Para os menos familiarizados com o tema, ilustramos sua importância mostrando como ela resolve a famosa recorrência de Catalan.

\section{RECORRÊNCIAS LINEARES DE COEFICIENTES CONSTANTES}

Uma sequência $\left(a_{n}\right)_{n \geq 1}$ é dita recorrente linear (de coeficientes constantes), se existirem um inteiro positivo $k$ e números complexos $u_{0}, \ldots, u_{k-1}$, nem todos nulos, tais que

$$
a_{n+k}=u_{k-1} a_{n+k-1}+\cdots+u_{0} a_{n},
$$

para todo inteiro $n \geq 1$.

O natural $k$ é denominado a ordem da recorrência linear e a equação (1) é a relação de recorrência ou, simplesmente, a recorrência satisfeita pela sequência. Neste caso, $\left(a_{n}\right)_{n \geq 1}$ é também denominada uma recorrência linear de ordem $k$.

Dados $\alpha_{1}, \ldots, \alpha_{k} \in \mathbb{C}$, é imediato verificar que há exatamente uma sequência $\left(a_{n}\right)_{n \geq 1}$ satisfazendo (1) e tal que $a_{j}=\alpha_{j}$, para $1 \leq j \leq k$. Portanto, uma recorrência linear de ordem $k$ fica totalmente determinada quando conhecemos a relação de recorrência linear por ela satisfeita e os valores de seus $k$ primeiros termos.

Se $\left(a_{n}\right)_{n \geq 1}$ satisfaz (1), definimos seu polinômio característico como o polinômio

$$
f(X)=X^{k}-u_{k-1} X^{k-1}-\cdots-u_{1} X-u_{0} .
$$

Nosso propósito, aqui, é dar uma demonstração elementar do seguinte resultado.

Teorema 2.1. Seja $\left(a_{n}\right)_{n \geq 1}$ uma sequência satisfazendo, para $n \geq 1$, a recorrência linear (1), onde $u_{0}, \ldots, u_{k-1}$ são números complexos dados, com $u_{0} \neq 0$. Sejam $z_{1}$, $\ldots, z_{l}$ as raízes duas a duas distintas do polinômio característico de $\left(a_{n}\right)_{n \geq 1}$, com multiplicidades respectivamente iguais a $m_{1}, \ldots, m_{l}$. Então, para $n \geq 1$ temos

$$
a_{n}=p_{1}(n-1) z_{1}^{n-1}+\cdots+p_{l}(n-1) z_{l}^{n-1},
$$

onde $p_{1}, \ldots, p_{l} \in \mathbb{C}[X]$ são polinômios de graus menores ou iguais a $m_{1}-1, \ldots$, $m_{l}-1$, respectivamente, os quais são totalmente determinados pelos valores de $a_{1}$, $\ldots, a_{k}$.

Em linhas gerais, a dinâmica da demonstração é a seguinte: inicialmente, mostramos que a série de potências

$$
F(z)=\sum_{n \geq 1} a_{n} z^{n}
$$

converge em um disco do plano complexo centrado em 0 . Em seguida, sendo $f$ o polinômio característico da sequência e $h(X)=-u_{0} X^{k}-u_{1} X^{k-1}-\cdots-u_{k-1} X+1$ seu recíproco, mostramos que

$$
h(z) F(z)=z p(z),
$$


para um certo polinômio $p \in \mathbb{C}[X] \backslash\{0\}$, de grau $\partial p \leq k-1$. Por fim, expandimos $\frac{z p(z)}{h(z)}$ em um disco centrado em 0 e de raio possivelmente menor, e aplicamos a (3) a unicidade da expansão em série de potências. Vejamos os detalhes.

Prova. Afirmamos inicialmente que existe uma constante $R_{0}>0$ tal que $\left|a_{n}\right| \leq R^{n}$, para todos $n \geq 1$ e $R>R_{0}$. De fato, se $\left|a_{n}\right| \leq R^{n}$ para $1 \leq n<m$, com $m>k$, então a desigualdade triangular fornece

$$
\begin{aligned}
\left|a_{m}\right| & \leq\left|u_{k-1}\right| R^{m-1}+\cdots+\left|u_{1}\right| R^{m-k+1}+\left|u_{0}\right| R^{m-k} \\
& =R^{m-k}\left(\left|u_{k-1}\right| R^{k-1}+\cdots+\left|u_{1}\right| R+\left|u_{0}\right|\right) .
\end{aligned}
$$

Portanto, se $g(X)=X^{k}-\left|u_{k-1}\right| X^{k-1}-\cdots-\left|u_{1}\right| X-\left|u_{0}\right|$ e $R_{0}>0$ for tal que $g(R)>0$ para $R>R_{0}$, então, para cada um de tais $R \mathrm{~s}$, temos pelos cálculos acima que $\left|a_{m}\right| \leq R^{m-k} \cdot R^{k}=R^{m}$. Basta, pois, escolhermos de início $R_{0}>0$ tal que $\left|a_{1}\right|, \ldots,\left|a_{k}\right| \leq R_{0}$ e $g(R)>0$, para todo $R>R_{0}$.

Uma vez que $\left|a_{n}\right| \leq R^{n}$ para todo inteiro $n \geq 1$ e todo $R>R_{0}$, o teste da comparação garante a convergência de $F$ no disco aberto $D\left(0 ; \frac{1}{R}\right)$ do plano complexo. Para mostrar que (3) vale em tal disco, temos primeiramente que

$$
\begin{aligned}
h(z) F(z)= & -\sum_{j=0}^{k-1} \sum_{n \geq k-j+1} u_{j} a_{n-k+j} z^{n}+\sum_{n \geq 1} a_{n} z^{n} \\
= & -\sum_{n \geq k+1} u_{0} a_{n-k} z^{n}-\sum_{n \geq k} u_{1} a_{n-k+1} z^{n} \\
& -\sum_{j=2}^{k-1} \sum_{n \geq k} u_{j} a_{n-k+j} z^{n}+\sum_{n \geq k} a_{n} z^{n} \\
& -\sum_{j=2}^{k-1} \sum_{n=k-j+1}^{k-1} u_{j} a_{n-k+j} z^{n}+\sum_{n=1}^{k-1} a_{n} z^{n} .
\end{aligned}
$$

Agora, segue de (1) que

$$
\begin{aligned}
& -\sum_{j=2}^{k-1} \sum_{n \geq k} u_{j} a_{n-k+j} z^{n}+\sum_{n \geq k} a_{n} z^{n}=\sum_{n \geq k}\left(-\sum_{j=2}^{k-1} u_{j} a_{n-k+j}+a_{n}\right) z^{n} \\
= & \left(-\sum_{j=2}^{k-1} u_{j} a_{j}+a_{k}\right) z^{k}+\sum_{n \geq k+1}\left(u_{0} a_{n-k}+u_{1} a_{n-k+1}\right) z^{n} .
\end{aligned}
$$


Daí,

$$
\begin{aligned}
h(z) F(z)= & -\sum_{n \geq k+1} u_{0} a_{n-k} z^{n}-\sum_{n \geq k} u_{1} a_{n-k+1} z^{n} \\
& +\left(-\sum_{j=2}^{k-1} u_{j} a_{j}+a_{k}\right) z^{k}+\sum_{n \geq k+1}\left(u_{0} a_{n-k}+u_{1} a_{n-k+1}\right) z^{n} \\
& -\sum_{j=2}^{k-1} \sum_{n=k-j+1}^{k-1} u_{j} a_{n-k+j} z^{n}+\sum_{n=1}^{k-1} a_{n} z^{n} \\
= & \left(a_{k}-\sum_{j=1}^{k-1} u_{j} a_{j}\right) z^{k}-\sum_{j=2}^{k-1} \sum_{n=k-j+1}^{k-1} u_{j} a_{n-k+j} z^{n}+\sum_{n=1}^{k-1} a_{n} z^{n},
\end{aligned}
$$

e basta escrever a última expressão acima como $z p(z)$.

Como $h(0)=1 \neq 0$, aumentando $R$, se necessário, podemos supor que $h(z) \neq 0$ para $z \in D\left(0 ; \frac{1}{R}\right)$. Por outro lado, sendo $z_{1}, \ldots, z_{l}$ as raízes complexas de $f$, com multiplicidades respectivamente iguais a $m_{1}, \ldots, m_{l}$, temos

$$
h(X)=\left(1-z_{1} X\right)^{m_{1}} \ldots\left(1-z_{l} X\right)^{m_{l}},
$$

de sorte que

$$
F(z)=\frac{z p(z)}{\left(1-z_{1} z\right)^{m_{1}} \ldots\left(1-z_{l} z\right)^{m_{l}}},
$$

para todo $z \in D\left(0 ; \frac{1}{R}\right)$.

Agora, como $p$ tem grau menor que o do denominador de (4), aplicando a fórmula de decomposição em frações parciais a (4), concluímos pela existência, para $1 \leq j \leq l$ e $1 \leq n_{j} \leq m_{j}$, de constantes $d_{j n_{j}}$, unicamente determinadas pelos coeficientes de $p$ (e, portanto, por $a_{1}, a_{2}, \ldots, a_{k}$ e $u_{0}, u_{1}, \ldots, u_{k-1}$ ), tais que

$$
F(z)=z \sum_{j=1}^{l} \sum_{n_{j}=1}^{m_{j}} \frac{d_{j n_{j}}}{\left(1-z_{j} z\right)^{n_{j}}},
$$

para todo $z \in D\left(0 ; \frac{1}{R}\right)$.

Para continuar, precisamos do seguinte fato, o qual pode ser mostrado facilmente a partir do caso $m=1$, por indução sobre $m$ ou por derivação termo a termo: se $a \in \mathbb{C} \backslash\{0\}$ e $m \in \mathbb{N}$, então

$$
\frac{1}{(1-a z)^{m}}=\sum_{n \geq 0}\left(\begin{array}{c}
n+m-1 \\
m-1
\end{array}\right) a^{n} z^{n}
$$

para todo $z \in D\left(0 ; \frac{1}{|a|}\right)$.

Em particular, se $r=\min \left\{\frac{1}{R}, \frac{1}{\left|z_{1}\right|}, \ldots, \frac{1}{\left|z_{l}\right|}\right\}$, então (6) permite escrever

$$
\frac{1}{\left(1-z_{j} z\right)^{n_{j}}}=\sum_{n \geq 0}\left(\begin{array}{c}
n+n_{j}-1 \\
n_{j}-1
\end{array}\right) z_{j}^{n} z^{n}
$$


para $1 \leq j \leq l, 1 \leq n_{j} \leq m_{j}$ e $z \in D(0 ; r)$. Portanto, voltando a (5) e para $|z|<r$, temos

$$
\begin{aligned}
F(z) & =z \sum_{j=1}^{l} \sum_{n_{j}=1}^{m_{j}} \sum_{n \geq 0} d_{j n_{j}}\left(\begin{array}{c}
n+n_{j}-1 \\
n_{j}-1
\end{array}\right) z_{j}^{n} z^{n} \\
& =\sum_{n \geq 1}\left(\sum_{j=1}^{l} \sum_{n_{j}=1}^{m_{j}} d_{j n_{j}}\left(\begin{array}{c}
n+n_{j}-2 \\
n_{j}-1
\end{array}\right) z_{j}^{n-1}\right) z^{n} .
\end{aligned}
$$

Por fim, uma vez que $a_{n}$ é o coeficiente de $z^{n}$ na série que define $F$, segue da última igualdade acima e da unicidade da expansão em série que

$$
\begin{aligned}
a_{n} & =\sum_{j=1}^{l} \sum_{n_{j}=1}^{m_{j}} d_{j n_{j}}\left(\begin{array}{c}
n+n_{j}-2 \\
n_{j}-1
\end{array}\right) z_{j}^{n-1} \\
& =\sum_{j=1}^{l} \sum_{n_{j}=1}^{m_{j}} \frac{d_{j n_{j}}}{\left(n_{j}-1\right) !}\left(n+n_{j}-2\right)\left(n+n_{j}-3\right) \ldots(n+1) n z_{j}^{n-1} \\
& =\sum_{j=1}^{l} p_{j}(n-1) z_{j}^{n-1},
\end{aligned}
$$

onde

$$
p_{j}(X)=\sum_{n_{j}=1}^{m_{j}} \frac{d_{j n_{j}}}{\left(n_{j}-1\right) !}\left(X+n_{j}-1\right)\left(X+n_{j}-2\right) \ldots(X+1) z_{j}^{n-1}
$$

é um polinômio de grau menor ou igual a $m_{j}-1$.

Concluímos esta seção apresentando uma aplicação interessante do Teorema 2.1, a qual também não é conhecida como deveria.

Exemplo 2.2. Dadas uma sequência $\left(a_{n}\right)_{n \geq 1}$ e um inteiro $m>1$, dizemos que $\left(a_{n}\right)_{n \geq 1}$ é uma

i. PA de ordem 1 se $\left(a_{n}\right)_{n \geq 1}$ for uma PA (progressão aritmética).

ii. PA de ordem $m$ se a sequência $\left(b_{n}\right)_{n \geq 1}$, tal que $b_{n}=a_{n+1}-a_{n}$ para $n \geq 1$, for uma $P A$ de ordem $m-1$.

Uma sequência $\left(a_{n}\right)_{n \geq 1}$ é uma PA de ordem $m$ se, e só se,

$$
\left(\begin{array}{c}
m+1 \\
0
\end{array}\right) a_{n+m+1}-\left(\begin{array}{c}
m+1 \\
1
\end{array}\right) a_{n+m}+\cdots+(-1)^{m+1}\left(\begin{array}{c}
m+1 \\
m+1
\end{array}\right) a_{n}=0,
$$

para todo $n \geq 1$. Esse fato pode ser facilmente demonstrado por indução sobre $m \geq 1$, com o auxilio da relação de Stifel entre números binomiais.

Se $\left(a_{n}\right)_{n \geq 1}$ é uma PA de ordem $m$, então (7) vale para todo $n \geq 1$, de sorte que o polinômio característico de $\left(a_{n}\right)_{n \geq 1}$ é

$$
\begin{aligned}
f(X) & =\left(\begin{array}{c}
m+1 \\
0
\end{array}\right) X^{m+1}-\left(\begin{array}{c}
m+1 \\
1
\end{array}\right) X^{m}+\cdots+(-1)^{m+1}\left(\begin{array}{l}
m+1 \\
m+1
\end{array}\right) \\
& =(X-1)^{m+1} .
\end{aligned}
$$


Assim, o Teorema 2.1 garante a existência de constantes $\alpha_{0}, \alpha_{1}, \ldots, \alpha_{m}$ tais que

$$
a_{n}=\alpha_{0}+\alpha_{1}(n-1)+\cdots+\alpha_{m}(n-1)^{m},
$$

para todo $n \geq 1$. Avaliando a relação acima para $n=1$, obtemos $\alpha_{0}=a_{1}$; avaliando-a para $n=2, \ldots, m+1$, obtemos $\alpha_{1}, \ldots, \alpha_{m}$ como soluções do sistema linear de equações

$$
\begin{cases}\alpha_{1}+\alpha_{2}+\cdots+\alpha_{m} & =a_{2}-a_{1} \\ 2 \alpha_{1}+2^{2} \alpha_{2}+\cdots+2^{m} \alpha_{m} & =a_{3}-a_{1} \\ \cdots & \\ m \alpha_{1}+m^{2} \alpha_{2}+\cdots+m^{m} \alpha_{m}= & a_{m+1}-a_{1}\end{cases}
$$

\section{A FóRMUla DE iNVERSÃo LAGRANGIANA}

Conforme veremos mais adiante, a modelagem de situações combinatórias interessantes, como o clássico problema de Catalan, traduz-se na busca de soluções analíticas $y=y(z)$ para uma equação da forma

$$
y=z \phi(y),
$$

em que $\phi: D(0 ; r) \subset \mathbb{C} \rightarrow \mathbb{C}$ é uma função analítica complexa satisfazendo $\phi(0) \neq 0$.

Uma tal solução $y(z)=\sum_{k>1} y_{n} z^{n}$ sempre existe num disco $D(0 ; s)$, para algum $s>0$, e a fórmula de inversão lagrangiana explicita $y_{n}$ em termos do coeficiente de $z^{n-1}$ na expansão em série de potências da função $z \mapsto \phi(z)^{n}$. Mais precisamente, temos o seguinte

Teorema 3.1 (Lagrange). Seja $\phi: D(0 ; r) \subset \mathbb{C} \rightarrow \mathbb{C}$ uma função analítica complexa tal que $\phi(0) \neq 0$, e $\phi(z)=\sum_{k \geq 0} \phi_{k} z^{k}$ sua expansão em série de potências em torno de 0 . Então, existe $s>0$ tal que, para $z \in D(0 ; s) \subset \mathbb{C}$, a equação $y=z \phi(y)$ admite uma única solução analítica $y(z)=\sum_{k \geq 1} y_{k} z^{k}$. Ademais,

$$
y_{n}=\frac{1}{n}\left[u^{n-1}\right]\left(\phi(u)^{n}\right)
$$

para todo inteiro $n \geq 1$, onde $\left[u^{n-1}\right]\left(\phi(u)^{n}\right)$ denota o coeficiente de $u^{n-1}$ na expansão de $u \mapsto \phi(u)^{n}$ centrada em 0 .

A ideia da prova é, primeiramente, utilizar o teorema da aplicação implícita para mostrar que (8) admite uma única solução continuamente diferenciável $y=y(z)$ numa vizinhança de 0 , com $y(0)=0$. Em seguida, mostramos que, vista com função complexa, y é analítica. Por fim, utilizamos a fórmula integral de Cauchy para obter (9). Vamos aos detalhes.

Prova. Consideremos a função $F: \mathbb{C} \times D(0 ; r) \rightarrow \mathbb{C}$ dada por $F(y, z)=y-z \phi(y)$. Identificando (da maneira usual) $\mathbb{C}$ com $\mathbb{R}^{2}, \mathbb{C} \times D(0 ; r)$ com um aberto de $\mathbb{R}^{4}$, e calculando a derivada de Fréchet $d_{y} F$ de $F$ com respeito a $y$, obtemos

$$
d_{y} F(y, z)=\mathrm{Id}-z \phi^{\prime}(y),
$$

onde $\phi^{\prime}(y)$ denota a derivada complexa de $\phi$ em $y$ e identificamos $z \phi^{\prime}(y)$ com um operador linear em $\mathbb{R}^{2}$, da maneira usual. 
Agora, tome $s>0$ tal que $\phi(y) \neq 0$ para todo $y \in D(0 ; s)$, e note que $F^{-1}(0)=$ $\{(y, z) ; F(y, z)=0\}$ é não vazio, pois contém pelo menos o ponto $(0,0)$. Então, em $F^{-1}(0) \cap(D(0 ; s) \times D(0 ; r))$, temos que

$$
d_{y} F(y, z)=\operatorname{Id}-\frac{\phi^{\prime}(y)}{\phi(y)} y .
$$

(Aqui, novamente vemos $\frac{\phi^{\prime}(y)}{\phi(y)} y$ como um operador linear em $\mathbb{R}^{2}$.)

Mas como $\lim _{y \rightarrow 0} \frac{\phi^{\prime}(y)}{\phi(y)} y=0$, podemos supor que $s>0$ foi tomado tão pequeno que $\left|\frac{\phi^{\prime}(y)}{\phi(y)} y\right|<1$ para $y \in D(0 ; s)$. Portanto, em $F^{-1}(0,0) \cap(D(0 ; s) \times D(0 ; r))$ temos

$$
d_{y} F(y, z)=\operatorname{Id}-T_{y},
$$

onde $T_{y}: \mathbb{R}^{2} \rightarrow \mathbb{R}^{2}$ é um operador linear de norma $\left\|T_{y}\right\|<1$.

Precisamos, agora, da afirmação a seguir, a qual é uma decorrência imediata do teorema do núcleo e da imagem.

Afirmação Se $T: \mathbb{R}^{p} \rightarrow \mathbb{R}^{p}$ é um operador linear com norma $\|T\|<1$, então o operador linear $S=\mathrm{Id}-T$ é invertível.

Pela afirmação, a derivada $d_{y} F(y, z): \mathbb{R}^{2} \rightarrow \mathbb{R}^{2}$ é um operador invertível, e o teorema da aplicação implícita ([4], capítulo 5) garante que, diminuindo $r$ e $s$ um pouco mais, se necessário, $F^{-1}(0)$ define o gráfico de uma aplicação diferenciável (real) $y=y(z): D(0 ; r) \rightarrow D(0 ; s)$, tal que $y(0)=0$; em particular, $y=y(z)$ é contínua.

Afirmamos que a função $y$ é injetiva em $D(0 ; r)$. De fato, para $z_{1}, z_{2} \in D(0 ; r)$, se $y\left(z_{1}\right)=y\left(z_{2}\right)=w$, então a relação $y(z)=z \phi(y(z))$ dá-nos

$$
z_{1} \phi(w)=z_{1} \phi\left(y\left(z_{1}\right)\right)=y\left(z_{1}\right)=y\left(z_{2}\right)=z_{2} \phi\left(y\left(z_{2}\right)\right)=z_{2} \phi(w) ;
$$

mas, como $\phi(w) \neq 0$ para $w \in D(0 ; s)$, segue que $z_{1}=z_{2}$.

Mostremos agora que $y$ é uma função analítica (complexa) de $z \in D(0 ; r)$ (isso é uma decorrência imediata da teoria de funções de várias variáveis complexas, mas daremos uma demonstração elementar, para tornar a exposição acessível a um público mais amplo). Se $z, z_{0} \in D(0 ; r)$, com $z \neq z_{0}$, então a injetividade de $y$ garante que $y(z) \neq y\left(z_{0}\right)$, e daí

$$
\begin{aligned}
\frac{y(z)-y\left(z_{0}\right)}{z-z_{0}} & =\frac{z \phi(y(z))-z_{0} \phi\left(y\left(z_{0}\right)\right)}{z-z_{0}} \\
& =\frac{z \phi(y(z))-z_{0} \phi(y(z))}{z-z_{0}}+\frac{z_{0} \phi(y(z))-z_{0} \phi\left(y\left(z_{0}\right)\right)}{z-z_{0}} \\
& =\phi(y(z))+z_{0} \frac{\phi(y(z))-\phi\left(y\left(z_{0}\right)\right)}{y(z)-y\left(z_{0}\right)} \cdot \frac{y(z)-y\left(z_{0}\right)}{z-z_{0}} .
\end{aligned}
$$

Logo,

$$
\frac{y(z)-y\left(z_{0}\right)}{z-z_{0}}\left(1-z_{0} \frac{\phi(y(z))-\phi\left(y\left(z_{0}\right)\right)}{y(z)-y\left(z_{0}\right)}\right)=\phi(y(z)) .
$$


Agora, a continuidade de $y$ fornece $\lim _{z \rightarrow z_{0}} y(z)=y\left(z_{0}\right)$, de sorte que

$$
\lim _{z \rightarrow z_{0}} z_{0} \frac{\phi(y(z))-\phi\left(y\left(z_{0}\right)\right)}{y(z)-y\left(z_{0}\right)}=\lim _{y(z) \rightarrow y\left(z_{0}\right)} z_{0} \frac{\phi(y(z))-\phi\left(y\left(z_{0}\right)\right)}{y(z)-y\left(z_{0}\right)}
$$

$$
=z_{0} \phi^{\prime}\left(y\left(z_{0}\right)\right) \text {, }
$$

o qual tende a 0 quando $z_{0} \rightarrow 0$. Podemos, então, supor adicionalmente que $r$ foi escolhido tão pequeno que $z_{0} \phi^{\prime}\left(y\left(z_{0}\right)\right) \neq 1 \mathrm{em} D(0 ; r)$. Portanto, fazendo $z \rightarrow z_{0} \mathrm{em}$ (10), concluímos que $y^{\prime}\left(z_{0}\right)=\lim _{z \rightarrow z_{0}} \frac{y(z)-y\left(z_{0}\right)}{z-z_{0}}$ existe e

$$
y^{\prime}\left(z_{0}\right)=\frac{\phi\left(y\left(z_{0}\right)\right)}{1-z_{0} \phi^{\prime}\left(y\left(z_{0}\right)\right)} .
$$

Em particular, $y^{\prime}(0)=\phi(0)=a_{0} \neq 0$ e $y$ é uma função analítica complexa de $z$, para $z \in D(0 ; r)$.

Se $y(z)=y_{0}+y_{1} z+\ldots+y_{n} z^{n}+\cdots$ é a expansão de $y$ em série de potências de $z$ em $D(0 ; r)$, então $y^{\prime}(z)=y_{1}+2 y_{2} z+\cdots+n y_{n-1} z^{n-1}+\cdots$. A fórmula integral da Cauchy (veja [6], por exemplo) dá-nos

$$
n y_{n}=\left[z^{n-1}\right]\left(y^{\prime}\right)=\frac{1}{2 \pi i} \int_{\gamma} \frac{y^{\prime}(z)}{z^{n}} d z,
$$

onde $\gamma:[0,2 \pi] \rightarrow D(0 ; r)$ é uma qualquer curva $C^{1}$ por partes tal que $\operatorname{Ind}(\gamma ; 0)=1$. Mas $y(z)=z \phi(y(z))$ fornece $z=\frac{y(z)}{\phi(y(z))}$, de maneira que

$$
n y_{n}=\left[z^{n-1}\right]\left(y^{\prime}\right)=\frac{1}{2 \pi i} \int_{\gamma} \frac{\phi(y(z))^{n} y^{\prime}(z) d z}{y(z)^{n}}=\frac{1}{2 \pi i} \int_{y \circ \gamma} \frac{\phi(w)^{n} d w}{w^{n}} .
$$

Por fim, como y é um difeomorfismo conforme sobre sua imagem, com $y(0)=0$, segue que

$$
\operatorname{Ind}(y \circ \gamma ; 0)=\operatorname{Ind}(\gamma ; 0)=1 .
$$

Assim, a igualdade acima nos dá, novamente por aplicação da fórmula integral de Cauchy,

$$
n y_{n}=\left[u^{n-1}\right]\left(\phi(u)^{n}\right)
$$

Exemplo 3.2. Dado um inteiro $n \geq 3$, o problema de Catalan consiste em calcular o número $a_{n}$ de maneiras distintas de particionar um poligono convexo de $n$ lados dado em triângulos, utilizando diagonais que não se intersectem no interior do polígono.

Pondo $a_{1}=0$ e $a_{2}=1$, é possivel mostrar (cf. o capitulo 3 de [2], por exemplo) que a sequência $\left(a_{n}\right)_{n \geq 1}$ satisfaz, para $n \geq 3$, a recorrência

$$
a_{n}=\sum_{k=1}^{n-1} a_{k} a_{n-k+1} .
$$

Fazendo $f(z)=\sum_{n \geq 0} a_{n+2} z^{n}$, um cálculo formal garante que (12) é satisfeita se, e só se,

$$
f(z)-1=z f(z)^{2} .
$$


Mas, com $y(z)=f(z)-1=\sum_{n \geq 1} a_{n+2} z^{n}$, a última relação acima é o mesmo que

$$
y=z(y+1)^{2} .
$$

Então, pondo $\phi(z)=(z+1)^{2}$, o Teorema 3.1 garante que (13) realmente admite uma única solução $y=y(z)$, analítica em um disco centrado em 0 e tal que

$$
a_{n+2}=\frac{1}{n}\left[u^{n-1}\right](u+1)^{2 n}=\frac{1}{n}\left(\begin{array}{c}
2 n \\
n-1
\end{array}\right)=\frac{1}{n+1}\left(\begin{array}{c}
2 n \\
n
\end{array}\right)
$$

para $n \geq 1$.

O exemplo anterior é uma pequena ilustração do uso da fórmula de inversão lagrangiana em Combinatória. Para muitas outras, veja a referência [3], onde os autores também aplicam a fórmula de inversão para obter estimativas assintóticas para os números de configurações possíveis em várias situações combinatórias.

\section{REFERÊNCIAS}

[1] T. Apostol. Calculus, Volume I. John Wiley \& Sons, Nova Iorque, 1967.

[2] A. Caminha. An Excursion Through Elementary Mathematics III. Discrete Mathematics and Polynomial Algebra. Springer Nature, Cham, 2018.

[3] P. Flajolet e R. Sedgewick. Analytic Combinatorics. Cambridge Univ. Press, Cambridge, 2009.

[4] E. L. Lima. Curso de Análise, vol. 2. IMPA, Rio de Janeiro, 2000.

[5] I. Niven. Formal Power Series. Amer. Math. Monthly 76 (1969), 871-889.

[6] M. Soares. Cálculo em uma Variável Complexa, Quinta Edição. SBM, Rio de Janeiro, 2016.

Universidade Federal do Ceará

Email address: caminha@mat.ufc.br 